\title{
Mouth cancer - What's it to you?
}

\author{
Mike Lewis, Guest Editor, \\ BDJ Mouth Cancer Themed Issue
}

The BDJ Upfront section includes editorials, letters, news, book reviews and interviews. Please direct your correspondence to the News Editor,

Adrian O'Dowd at BDJNews@nature.com. Press releases or articles may be edited, and should include a colour photograph if possible.

I was absolutely delighted when I was asked if I would be willing to act as Guest Editor for an issue of the $B D J$ that was to be themed on the subject of mouth cancer. Acceptance of this request has allowed me to not only bring together a collection of articles on a wide range of aspects of mouth cancer with direct relevance for the readership of the journal but also, through this editorial, it has given me an opportunity to express some of my personal views on one of the most important diseases within the specialty of dentistry. I hope that some of my observations related to mouth cancer, which are presented below, will prompt some discussion on the topic within the dental profession.

There are many issues related to my clinical role as a consultant in oral medicine that are challenging, such as the management of orofacial pain or extensive mucosal disease, but the one part of the job that I believe it is my duty to handle most sensitively is when I have to inform a patient that they have cancer. I have found over the years that this conversation impacts on the patient in contrasting ways. For some it represents a bombshell out of the blue whilst for others it is something that they had already strongly suspected may have been present. Either way, it is essential that the patient is supported fully from that point onwards, which will involve an introduction to a Macmillan nurse and attendance at a Head and Neck Oncology Multi-Disciplinary Team (MDT) clinic. The MDT encompasses a range of healthcare experts who can discuss all aspects of the cancer with the patient so that treatment decisions can be made in an informed and supportive way.

Whenever a patient under my consultant care is diagnosed with mouth cancer, I inevitably ask myself: 'Could it have been detected when smaller?' I use the word 'smaller' rather than 'earlier' deliberately. Unfortunately, the majority of cancers when first detected are greater than $2 \mathrm{~cm}$ in diameter. Size is extremely important in relation to mouth cancer since the prognosis is greatly improved if the cancer is found when less than $2 \mathrm{~cm}$ in diameter. Mouth cancer behaves differently in each individual case; some tumours develop slowly over many months whilst others grow rapidly within a few weeks. However, 'small' when diagnosed is key rather than how long the cancer has been present.

When pre-existing mucosal changes are involved, as in the case of potentially malignant disorders, it is impossible to determine when malignant transformation may occur. In my own experience, on the rare occasions that a cancer has developed in the many cases of lichen planus that I have profession in situations where a dental healthcare professional is found to have missed a cancer. Once diagnosed, the benefit of hind sight is all well and good but I believe that the very nature of the varied clinical presentation of mouth cancer lends itself to the potential to be missed. It is all too easy for some individuals to point the finger of blame without knowing all the facts. I fully accept of course that cancer should not be missed. I know personally that I have biopsied a mucosal abnormality on occasions that I would not have thought to be a cancer only to find when the pathology report is available that it was in fact a tumour. Whilst the clinical presentation is often highly suggestive of malignancy, particularly if there is induration, sometimes it is not so obvious. However, this aspect of

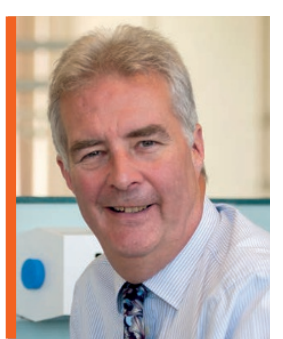

\section{'Any persistent abnormality, that has no obvious cause, must be investigated...'}

managed over the years, I ask myself: Did I miss something at the previous review? My observation with regard to the development of cancer in lichen planus is that, when it happens, it happens rapidly. So what is the optimal review period for patients with potentially malignant disorders? I do not believe that we actually know the answer to that question. I do what I feel best for the individual patient, taking into account all factors that may influence malignant transformation. However, I also always ask the patient to contact me before the time of their review date if they feel that something is 'changing' within their mouth.

I have been concerned in the past by an element of blame culture existing within the mouth cancer just confirms the need to apply the 'rule' that any persistent abnormality, that has no obvious cause, must be investigated. In practical terms this will mean a referral from primary care to an oral medicine or oral and maxillofacial surgery clinic. The increasing number of urgent suspected cancer (USC) referrals to secondary care is now impacting on the hospital services. I personally ensure that, as the consultant, I see all the patients on my NHS oral medicine clinics, which now regularly have more than 30 patients booked per session. Inevitably, USC patients are often added to the list at short notice since the 'two week rule' has to apply to this type of referral. In addition, I continually have to make a decision with regard to the urgency of 
an appointment from the written information provided on routine referrals. I must say that some requests contain minimal information and I am then concerned about the possibility of delaying the diagnosis of a potential cancer patient. The introduction of electronic referral systems may help in this regard since prompts for key clinical information can be included in the submission forms and improve communication. Fortunately, only around 5\% of USC referrals are subsequently found to be cancer, meaning that most patients have benign conditions.

The majority of cases of mouth cancer are squamous cell carcinoma, which represents out of control epithelial cell replication of the lining of the mouth. This surface change should be detectable by the eye. Do we all examine the tissues thoroughly enough at every visit? A mucosal abnormality that is $2 \mathrm{~cm}$ in diameter equates to the size of a thumb nail. Would a dental care professional take more time to examine the soft tissues for such an area of change if there was a finder's fee payment for mouth cancer? This is obviously an extremely controversial thought and I suspect not professionally acceptable!

When I started my oral medicine training more than three decades ago there were approximately 3,500 new cases of mouth cancer in the UK per year, usually affecting males over the age of 55 years. The number of new cases of mouth cancer diagnosed in the UK per has now risen to almost 8,000 cases per year, a doubling of numbers in my working life time. Not only am I seeing mouth cancer more frequently but the incidence in females is now equal to men and patients are significantly younger. These trends in the epidemiology of mouth cancer are worrying. On a positive note, the significant advance in the treatment of mouth cancer, particularly the use of free tissue flaps and dental implants, has greatly enhanced the quality of life for cancer patients. However, the overall five-year survival for mouth cancer post treatment is not improving and remains at only $50 \%$.

The scientific literature and popular press both use the terms oral cancer and mouth cancer. Which is correct? Both are acceptable but I must say that my preference is mouth cancer since I believe it is a more descriptive and inclusive terminology that is also understandable by the general public. Whilst on the subject of terminology, I would also like to promote the use of term abnormality rather than lesion. The word lesion comes from the Latin for injury. Describing a change within the orofacial tissue as abnormality is more accurate than using a term that implies an injury.
As I was writing this editorial, I became aware that a BBC Radio 2 presenter has just informed his listeners that he is going to need to take time off from work to undergo treatment for 'tongue cancer and removal of lymph nodes in his neck'. His subsequent tweet about this and the inclusion that it had been caught early, received 16k 'likes'. This media attention will hopefully raise awareness of mouth cancer within the general population and influence individuals to either check their mouths themselves or ask for an assessment by a dental professional.

Finally, I am extremely grateful to my professional colleagues who accepted my invitation to contribute articles to the Journal so enthusiastically. I hope that this themed issue contains something for everyone. I am particularly pleased that a number of the papers describe aspects of mouth cancer that are relevant to dental primary care. I requested that the journal was published in November to coincide with Mouth Cancer Awareness Month in the UK, a time that the dental profession has a specific focus on mouth cancer. I think that I have provided some impression of what mouth cancer means to me. I hope that the information presented within this $B D J$ themed issue inspires you to also think about what mouth cancer actually means to you. DOI: $10.1038 /$ sj.bdj.2018.980 